\title{
Low birth weight was associated with non-fatal coronary artery disease and stroke in nurses
}

\author{
Rich-Edwards JW, Stampfer MJ, Manson JE, et al. Birth weight and risk of cardiovascular disease in a cohort of women followed up \\ since 1976. BMJ 1997 Aug 16;315:396-400.
}

\section{Objective}

To determine if an association exists between birth weight and non-fatal cardiovascular disease in women who were nurses.

\section{Design}

Cohort study of women in the Nurses' Health Study followed up every 2 years from 1976 to 1992.

\section{Setting}

USA.

\section{Participants}

70297 women (mean age $42 \mathrm{y}$ in 1976) born between 1921 and 1946 , all registered nurses, who were free from coronary heart disease and stroke in 1976.

\section{Assessment of risk factors}

Women were grouped by their birth weight category: $<2268 \mathrm{~g}$ $(\mathrm{n}=1392), 2268-2495 \mathrm{~g}(\mathrm{n}=3549),>2495-3175 \mathrm{~g}(\mathrm{n}=21171)$, $>3175-3856$ g $(\mathrm{n}=31216),>3856-4536$ g $(\mathrm{n}=7723)$, or $>4536 \mathrm{~g}(\mathrm{n}=1638) .3608$ women who were born prematurely were not included in the analysis. Postal questionnaires were used to gather data on past and current cardiovascular disease risk factors in 1976 and birth weight in 1992.

\section{Main outcome measures}

Data on non-fatal myocardial infarction (symptoms and either electrocardiographic changes or increased cardiac enzyme concentrations), coronary revascularisation (coronary artery bypass grafting or angioplasty), and non-fatal stroke (evidence of a typical neurological deficit of sudden or rapid onset that persisted for $>24$ hours) that occurred between 1976 and 1992 were obtained from medical records and reviewed by doctors who were blind to the risk factors.

\begin{abstract}
Main results
Multivariate analysis controlled for age, body mass index, smoking, hypertension, cholesterol concentration, parental history of myocardial infarction at $<60$ years, diabetes, menopausal status, and use of postmenopausal hormones. Among women who were full term singletons, those who had birth weights $>2268-2495 \mathrm{~g}$ had an increased risk of coronary revascularisation (relative risk [RR] 1.61, 95\% CI 1.13 to 2.28) compared with those who had birth weights $>3175-3856 \mathrm{~g}$. Those who had birth weights $<2268 \mathrm{~g}$ had an increased risk of non-fatal stroke (ischaemic, haemorrhagic, and other strokes) (RR 2.29, CI 1.34 to 3.90) compared with those who had birth weights $>3175-3856 \mathrm{~g}$. There was an inverse trend towards birth weight and all types of non-fatal coronary heart disease (which included non-fatal myocardial infarction and coronary revascularisation) ( $\mathrm{p}$ for trend $=0.036)$, coronary revascularisation alone ( $\mathrm{p}$ for trend $=0.006$ ), all types of non-fatal strokes ( $p$ for trend $=0.002$ ), ischaemic stroke alone ( $\mathrm{p}$ for trend $=0.008$ ), and haemorrhagic stroke alone ( $\mathrm{p}$ for trend $=0.015$ ).
\end{abstract}

\section{Conclusion}

An inverse trend existed between birth weight and risk of non-fatal coronary artery disease and non-fatal stroke (ie, the lower the birth weight, the higher the risk of non-fatal coronary artery disease and non-fatal stroke) in nurses who were born full term.

Sources of funding: National Institutes of Health.

For article reprint:DrJ W Rich-Edwards, Department of Ambulatory Care and Prevention, Harvard Pilgrim Health Care and Harvard Medical School, 126 Brookline Avenue, Suite 200, Boston, MA 02215, USA. Fax +16174212763

\section{Commentary}

Limited research on cardiovascular disease in women has been done despite the fact that cardiovascular disease is the main cause of mortality for women in industrial nations. The study by Rich-Edwards et al gives attention to birth weight as a marker of non-fatal cardiovascular disease including myocardial infarction, coronary revascularisation, and stroke.

In this substudy of the Nurses' Health Study, the authors are thorough in their description of the purpose, design, data collection, data analysis, and limitations, which helps to establish credibility and context for the findings. They caution the reader in the interpretation of the data, noting that information was missing on several factors that affect birth weight; some misclassification of self reported birth weight existed; no data existed for 723 deaths caused by cardiovascular disease between 1976 and 1992; and several environmental and genetic factors that determine birth weight may have influenced cardiovascular risk. They noted that birth weight is related to adult cardiovascular risk primarily through indirect pathways rather than a direct causal link.

An association between birth weight and socioeconomic group would be expected, yet the inverse relation of birth weight and risk of cardiovascular disease remained after adjustment for ethnicity, socioeconomic group at age 16 , and husband's education. Because of the nature of the population, ethnic groups were not delineated or subanalysed to look at the strength of the relation between birth weight and cardiovascular disease in different groups. This would be of particular interest because African-American women have a greater risk of coronary artery disease mortality than whites, with a more pronounced incidence in lower socioeconomic classes. ${ }^{1}$ The other important note made by the authors is that the association was driven by extreme birth weights and that there was little variation among the $88 \%$ of women who had birth weights $>2495 \mathrm{~g}$.

The results of the study are relevant to all nurses who are involved in the prevention and management of cardiovascular disease in women. The findings increase our knowledge of the implications of birth weight as a marker of cardiovascular risk and the need to record birth weight and its context in the assessment and care process.

Donna Meagher-Stewart, RN, MHSc Professor, School of Nursing, Dalhousie University Halifax, Nova Scotia, Canada

1 Keller C, Fleury J, Bergstrom D. Risk factors for coronary heart disease in African-American women. Cardiovasc Nurs 1995;31:9-15. 